\title{
Radio-Star Scintillations From Ionospheric Waves
}

\author{
James W. Warwick
}

\author{
Contribution from Department of Asstrophysics and Atmospheric Physics, University of Colorado, and High \\ Altitude Observatory, Boulder, Colo.
}

(Received August 8, 1963)

\begin{abstract}
A swept-frequency interferometer operating in the frequency range from 7.6 to 41 megacycles per second records positions and durations of Cassiopeia A scintillations. Besides demonstrating the broad bandwidth characteristic of scintillations in this frequency range, the interferometer often reveals systematic trends in the duration and the position of the radio star as functions of frequency. On several occasions, the effects can be interpreted simply and directly in terms of wavelike irregularity structure at about 200 kilometers elevation. In one instance studied in detail, the wavelength was 17 kilometers from crest to crest and the phase velocity of the order of 60 meters per second. From a statistical argument the conclusion is drawn that most of the irregularities producing scintillations recorded by us are in motion towards the south. The absence of duration and angle-of-arrival effects from many other broadband scintillations implies the existence of a finer structure, superposed on the basic long-wavelength pattern.
\end{abstract}

\section{i. Introduction}

Radio-star scintillations arise from irregularities in the electron density of the ionosphere. Different regions of a plane-wave front impinging on the ionosphere undergo relative phase retardations and advances corresponding to the fluctuating electron density. If the fluctuations are thin, weak, and randomly distributed, the phase variations along the ionosphere may follow the same distribution as the electrons. However, a thick region of large fluctuations may produce phase variations dissimilar to the electron distribution. Therefore, any direct evidence that scintillations may provide on the nature of the irregularities is very welcome.

Wild and Roberts [1956] showed in Australia that Cygnus A scintillations from 40 to $70 \mathrm{Mc} / \mathrm{s}$ often extended over a broad bandwidth, indicating that the source of scintillations lay in focusing effects from ionospheric lenses and prisms. On the other hand, observations at single frequencies, or multiple frequencies at widely spaced intervals, usually are interpreted in terms of the random superposition of waves from several or many independent irregularities [Booker, 1958; Little et al., 1962 ; Lawrence et al., 1961]. Whether the difference in interpretations results from the Australian's geographic position, low-frequency spectral range, or dynamic spectral technique alone is still obscure. In the latter case, a thorough revision of standard scintillation theory may be required.

The Boulder spectrographic results confirm the broad bandwidths, in general $2: 1$ and often greater in the frequency range 7.6 to $41 \mathrm{Mc} / \mathrm{s}$. The majority of scintillations seen with this equipment do not show systematic tendencies of duration or position to vary with frequency or time. There is still, however, left over a considerable number of scintillations, greater than 10 percent of the total, which exhibit interesting patterns of motion and duration. The intent of this report is to show how this special type of scintillation can be interpreted by means of wave structure in the middle or upper ionosphere.

The Boulder swept-frequency interferometer is also used for observations of the sun and the planet Jupiter. Papers describing its operation in these fields have appeared in the literature on several occasions [Boischot et al., 1960; Warwick, 1961, 1963]. In addition, a summary article devoted to the special features of the receiver is currently in press [Lee and Warwick, 1963].

\section{Description of the Observations}

Figures 1, 2, and 3 illustrate complete examples of the particular type of scintillation under discussion. These are focused scintillations, for which the duration of the scintillation is least at an intermediate frequency, and increases towards both lower and higher frequencies. Sometimes, as shown in figures 2 and 3 , and perhaps in figure 1 as well, the scintillation forks into two branches at low frequencies, below the frequency of minimum duration. The fork rarely appears at high frequencies. The normal diurnal drift of the radio star fringes in the time-frequency domain is sketched-in on the record of figure 1. Compared with that drift, the drift of the radio star is relatively fast at high frequencies, and slow at low frequencies. At a given frequency, the relative motion is monotonic throughout the scintillation, and may or may not be coupled to an overall gross displacement of the position of the radio star from normal.

Figure 4 illustrates the geometry of the fringe and horizon for the scintillation pictured in figure 1 . Figure 4 includes the magnetic field orientation at a distance of $347 \mathrm{~km}$, and the cardinal directions at that point of the ionosphere, as seen in projection. 


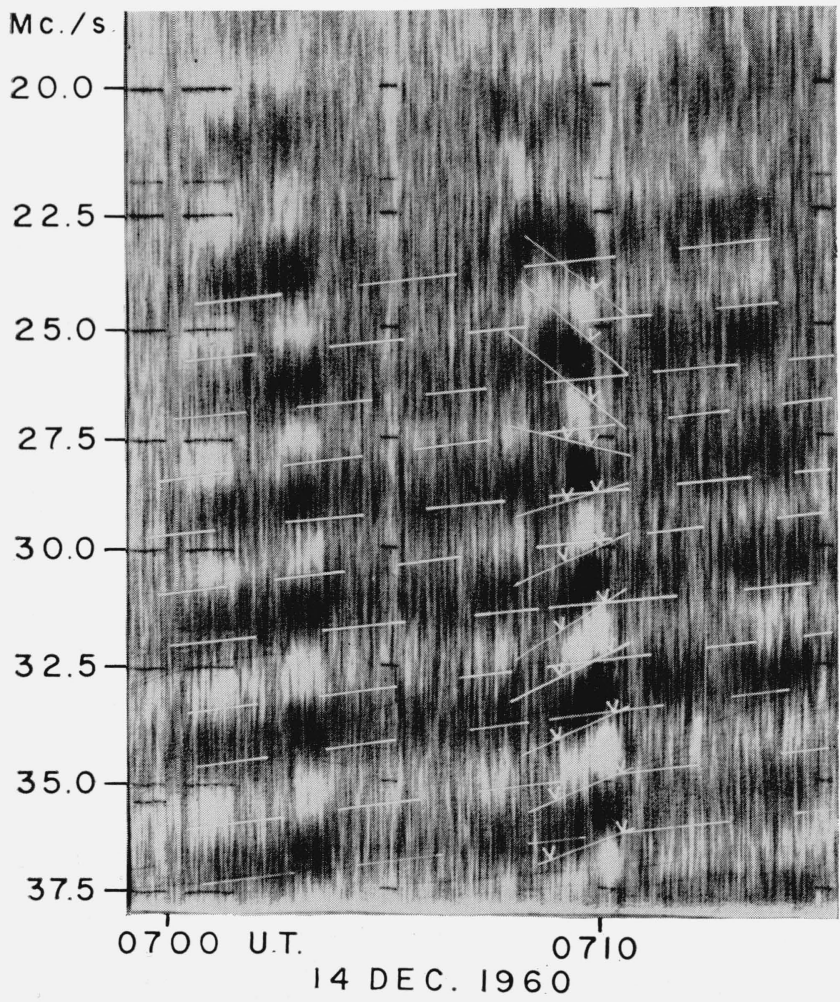

FIGU RE 1. Dynamic spectrum of a large focused scintillation.

The interferometer fringes produced during the diurnal motion of the radio star, Cassiopeia A, are slightly inclined, upwards to the right. They are indicated by dashed lines. The relatively tilted fringes during the strong scintillation at 0710 UT are indicated by line segments, on which are also indicated the apparent beginning and ending times of the event. These data are replotted in figures 8 and 14 .

The relative shift of the radio star is consistent with an angle-of-arrival pattern moving to the north (or east) along the almucantor at high frequencies, and to the south (or west) at low frequencies. The fringe shift-pattern, if it indeed represents a total motion that lies along the almucantor, is almost at right angles to the projected direction of the magnetic field.

\section{Interpretation of Motions and Duration as Functions of Frequency}

The majority of scintillations that show motions at all follow the pattern of shifts as described above. Although the coverage of our observations of Cassiopeia $\mathrm{A}$ is not complete in local or sidereal time a wide range of hour angles and local times is represented. For simplicity we speak of the irregularity motion as a wind. We shall show that the wind, if it is assumed to blow from one direction only, can explain the consistent fringe shift-pattern, independent of geographical position (sidereal time) or time of day (local time). Figure 5 represents the diurnal track of Cassiopeia A through the interferometer fringe pattern, and shows, like figure 4 , the cardinal directions and the magnetic field at several points along the track. The scintillation will not displace

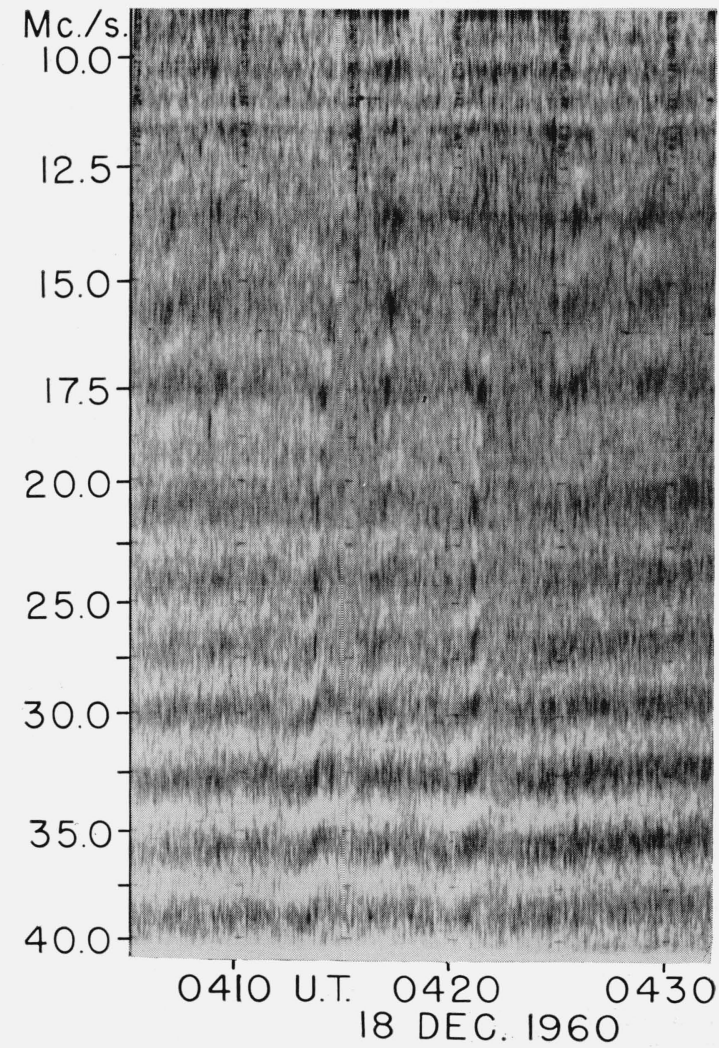

FIGURE 2. Dynamic spectrum of a large focused scintillation showing fork.

The scintillations at 0413 and 0421 UT both show the systematic departure of the angle of arrival from the diurnal trend as a function of frequency. The event at 0421 Universal Time also splits into two parts of roughly equal intensity below $20 \mathrm{Mc} / \mathrm{s}$. This effect is called a "fork."

the source observably if the wind moves the irregularity along the fringe; if the wind blows in other directions, the observed motion increases as the sine of the angle $\beta$ between the fringe and the projected direction of the wind vector, $\mathbf{v}$. The angular motion increases also as the sine of the angle $\alpha$ between $\mathbf{v}$ and the line of sight. For a given magnitude $v$ of the wind velocity, the apparent drift of the irregularity is greatest in or near the zenith. When the irregularity is distance $a$ from the observer, the angular shift depends on $v / a$. The angular drift of the irregularity in radians per second is therefore

$$
W=(v / a) \sin \alpha \sin \beta .
$$

The sign of $W$ depends on the direction of the component of $\mathbf{v}$ perpendicular to the fringe at the position of the radio star. Conventionally, we regard $W$ as negative for motions towards the south end of our interferometer's baseline, and positive towards the north.

Figure 6 illustrates the behavior of $W$ according to (1), on the assumption that the wind blows in the horizontal plane at an altitude of $178 \mathrm{~km}$ with a constant speed $v=60 \mathrm{~m} / \mathrm{s}$. $W$ is parametric in the angle $\phi$, the azimuth towards which the wind blows. 


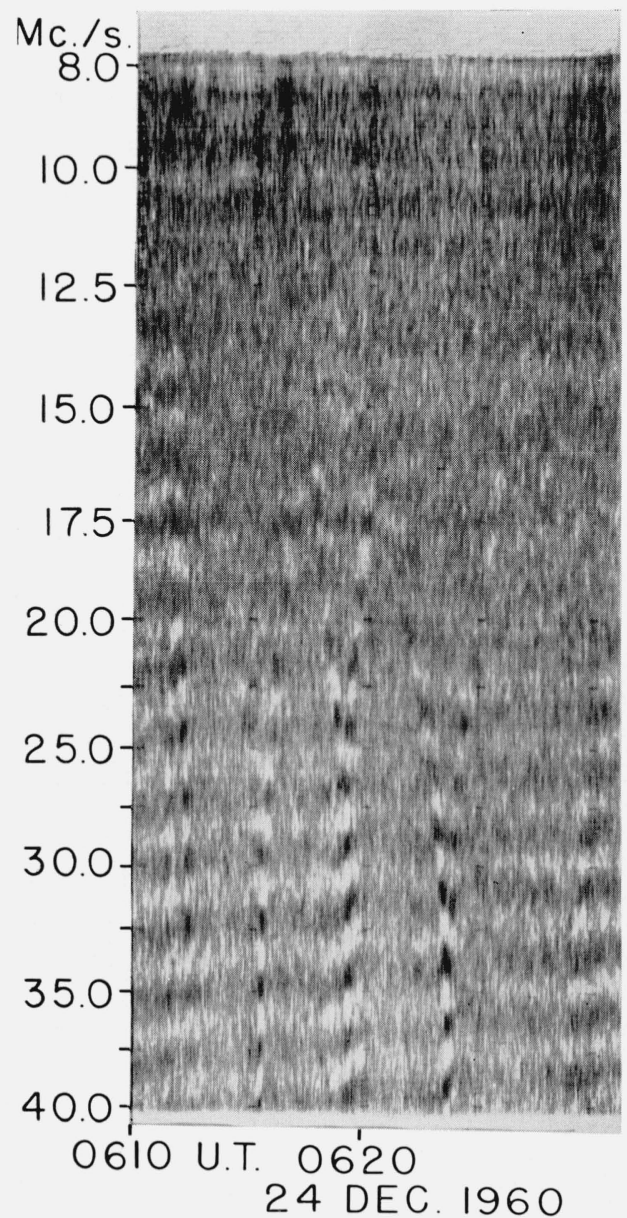

Figure 3. Dynamic spectra of focused scintillations showing periodicity of occurrence and different positions of forks from event to event.

The pattern of angle of arrival variation during the scintillations is the same as ound for the other scintillations in figures 1 and 2 . The event at 0612 UT may be forked on the high-frequency side. The events at 0615,0619 , and 0624 UT fork respectively at 32,27 , and $35 \mathrm{Mc} / \mathrm{s}$. The source virtually disappears between each of the four successive scintillations at five minute intervals.

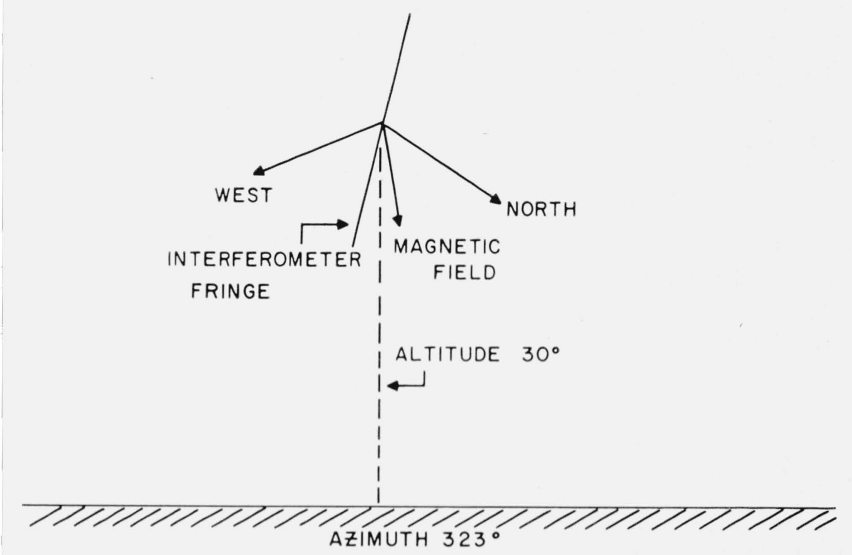

Figure 4. Fringe, magnetic field, and horizon for the scintillation of figure 1.

The magnetic field, north, and west are drawn for a computed distance of 347 $\mathrm{km}$ to the source of the scintillation (see text).
We show below that the actual pattern of drift as a function of frequency corresponds to negative values of $W$. From figure 6 one sees that negative values occur, outside of sidereal times $10^{h}$ to $16^{h}$, for wind vectors from about $130^{\circ}$ to $310^{\circ}$. In the interval $10^{h}$ to $16^{h}$, negative values occur from $210^{\circ}$ to about $10^{\circ}$. Owing to the great distance to the ionosphere at these times, $W$ is small. The largest negative values of the drift occur for wind vectors in the quadrant from $150^{\circ}$ to $240^{\circ}$. The preferred wind direction mentioned above is therefore roughly towards the southern quadrant of the sky.

The observed phenomena indicate that an equivalent ionospheric lens focuses radio star energy onto our receivers. Figure 7 shows a cross section of a biconcave lens surface enclosing a region of enhanced electron density, for several different focal lengths corresponding to different frequencies of observation. In figure $7 \mathrm{a}$, the focus lies at ground level. Rays from all points on the lens contribute to the signal observed at a given point. If the image of the radio star extends over an appreciable area, then motions of the star can be measured at the focus, as the sum of the diurnal and wind-drift motions. In figure $7 \mathrm{~b}$, the focus lies below ground level, and corresponds to observations made at a higher frequency than those of figure 7a. Rays from only a limited region of the lens contribute to the signal observed by the interferometer, in fact just the rays spanned by the baseline of our equipment. The diurnal motion and ionospheric drift combine to produce an apparent drift indicated by arrows in figure 7 ; this apparent drift is the angular swing of the line connecting the radio star to the lens. Figure $7 \mathrm{~b}$ demonstrates that for high frequencies the measured drift takes place in the opposite sense to the apparent drift. As shown in figure $7 \mathrm{c}$, for low frequencies the measured drift lies in the same direction as the apparent drift of the ionospheric lens.

In the swept-frequency interferometer used for these observations the tilt of the fringes determines whether an apparent fringe shift moves the source towards or away from the electrical phase center of the system. For identical antennas, with identical lead-in lines to the receiver, this electrical phase center would be the perpendicular bisector plane of the interferometer baseline. In actuality, the phase center is tipped towards one or the other end of the baseline, and therefore describes a cone instead of a plane. This cone, the collimation cone, varies from one observation period to another in a pre-established pattern. In the present observations, the fringe shifts at low frequency indicate the source is moving towards the collimation cone, that is, towards the southern end of the baseline. The wind therefore is blowing towards the south.

It should be remarked that for the scintillations shown in figures 1,2 , and 3 the diurnal motion lies roughly parallel to the fringe direction. Most of the observed drift therefore results from ionospheric winds rather than motion of the radio star along its diurnal track. 


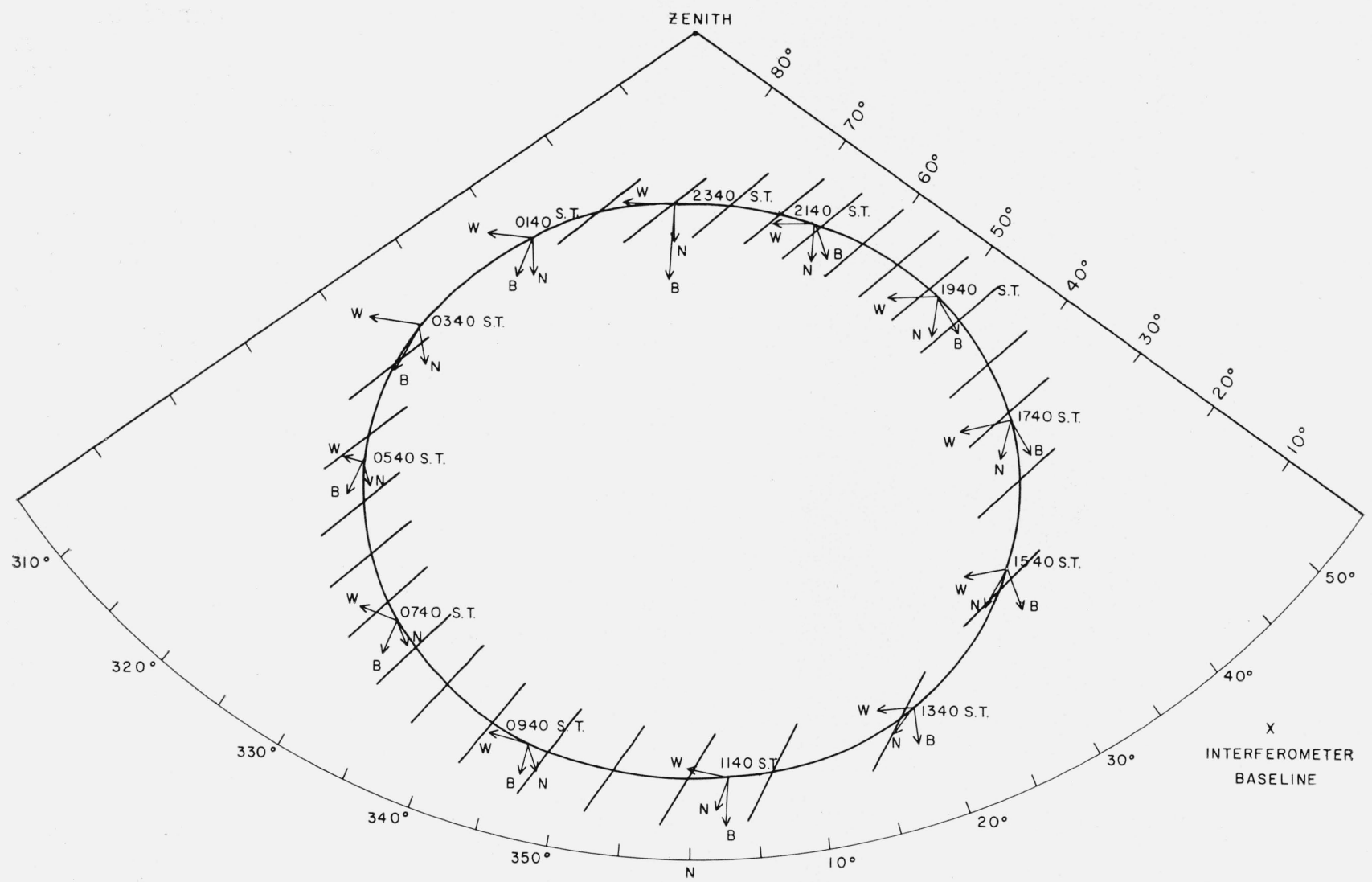

Figure 5. Diurnal path of Cassiopeia $A$ in the Boulder horizontal coordinate system.

$\mathrm{W}, \mathrm{N}, \mathrm{B}$ represent the western direction, the northern direction, and the magnetic field direction at an altitude of $178 \mathrm{~km}$. The line segments crossing the diurnal track are portions of the interferometer fringe system. Angles are measured correctly relative to the fringes as drawn but are distorted relative to the projection system track are portions of the interferometer fringe system. Angles are measured correctly relative to the fringes as drawn but are
used for the diurnal track and fringe system. Sidereal times are indicated around the track; figure 1 occurred at 0540 ST.

If refraction effects in a lens correctly explain the minimum duration of the scintillation at an intermediate frequency, we expect to find a linear relation between duration and the square of the reciprocal frequency. Figure 8 shows the observed relation for the data of figure 1 , where the duration minus the minimum duration is plotted rather than the duration itself. The minimum occurred near 27.5 $\mathrm{Mc} / \mathrm{s}$ and was 39 seconds. This subtraction is justified because of the angular size of the radio star, because of the diffractive limitation on sharpness of focus, and because irregularities on the lens surface spread the image.

\section{Forked Scintillations}

When the preceding study of lenses as a source of scintillations was begun, it seemed plausible to interpret the forked effect as the result of a geometrical-optic caustic, which occurs typically in association with out-of-focus images. Figure 9 illus- trates the geometry of such a caustic for a spherical lens. Since outer parts of the lens bend rays through larger angles than inner parts, the intersection points of adjacent rays at the edge of the lens lie closer to the lens than the intersections of rays near the center. The figure represents the caustic formed at a fixed frequency. At high frequencies, the curve is similar, but shifted away from the lens. The effect brings the widely spread portions of the caustic to ground level at high frequencies. The spherical lens assumption then cannot be generally correct if, indeed, the forking results from a caustic at all.

In the ionosphere, a cylindrical lens would occur as a result of a columnar electron density structure. Enhanced electron density with cylindrical symmetry in localized regions might, as suggested in figure 10 , lead to convergent lenses, although not with the proper caustic properties. The root cause of these difficulties appears to be the multiplyconnected structure of the isoelectronic surface. This leads to discontinuities in the geometrical-optic behavior of the ionosphere. 


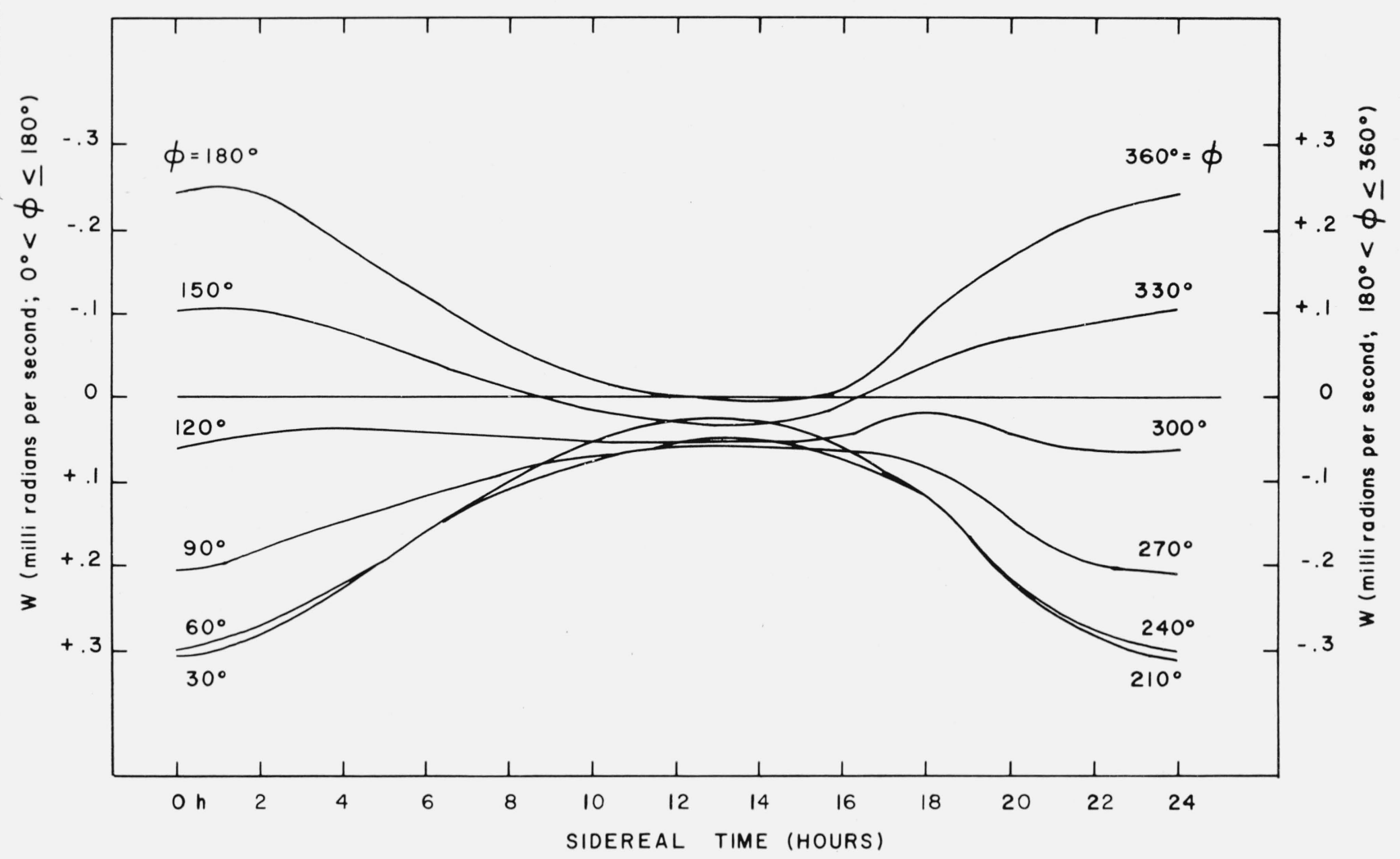

Figure 6. Was a function of sidereal time and wind direction.

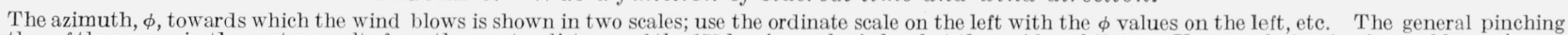

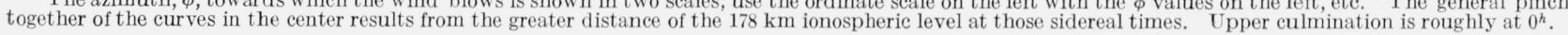

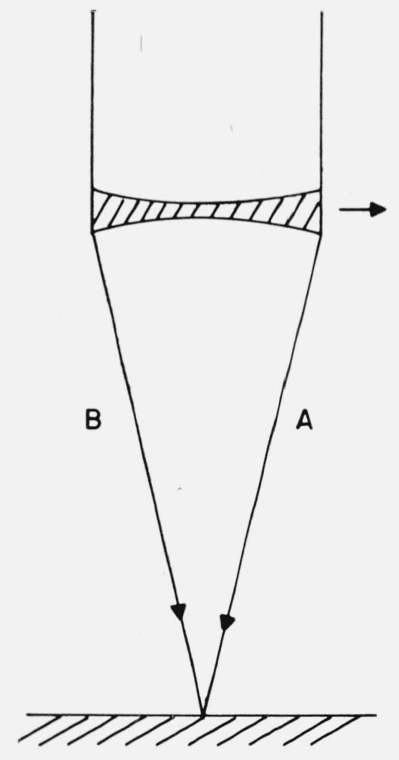

(a)

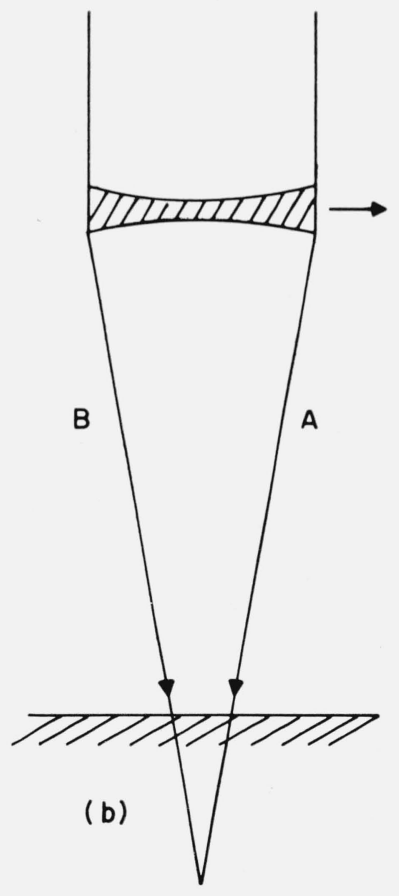

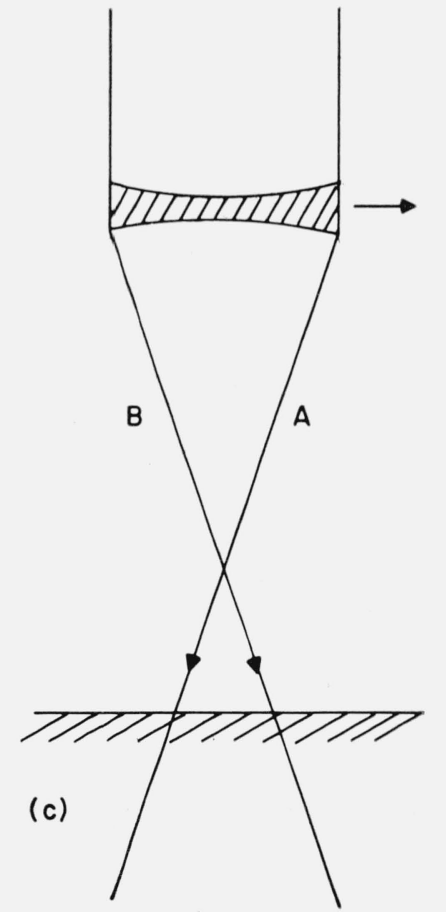

Figure 7. Schematic geometry of focusing action from a moving lens.

a. Rays $A$ and $B$ come to a focus at the ground, at a central frequency.

b. Rays $A$ and $B$ focus below the ground, at a high frequency.

c. Rays $A$ and $B$ focus above the ground, at a low frequency. 


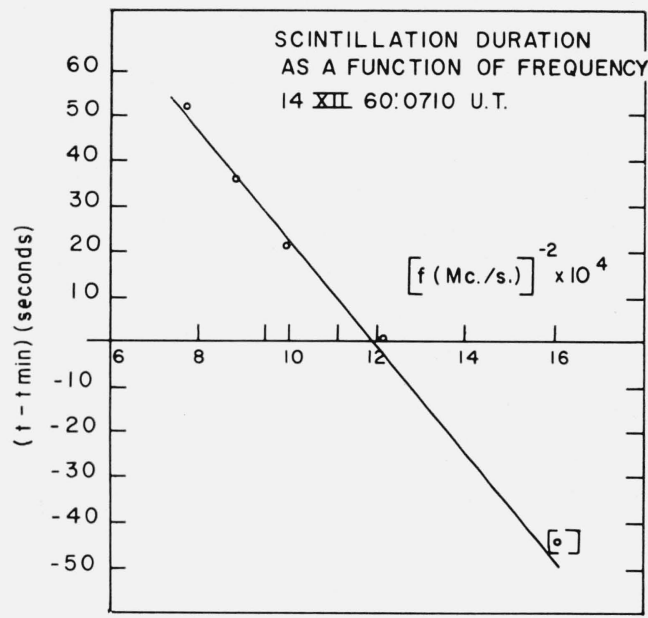

Figure 8. Scintillation duration as a function of the inverse square of the frequency.

Event of figure 1, 14 December 1960 at 0710 UT. A point is plotted for one frequency below the frequency of minimum duration, where in fact some confusion may,exist with an earlier weak scintillation. There is a possibility that the scintillation is forked at low frequencies, like the scintillations shown in figures 2 and 3 . The point is included since it is apparently consistent with the obviously linear trend of the observations at higher frequencies.

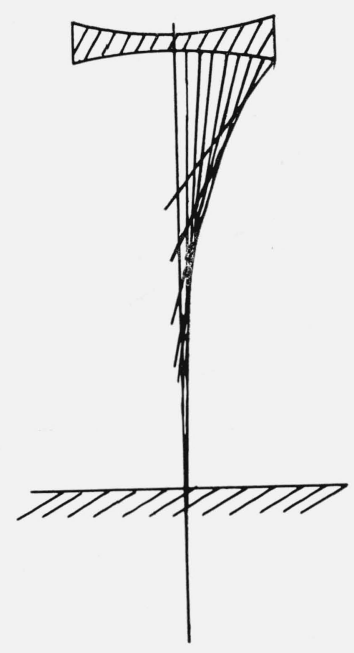

FIGURE 9. The caustic formed by a spherical converging lens lies inside the region of best focus.

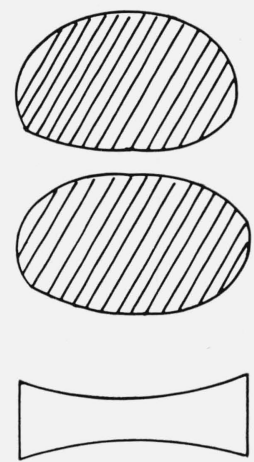

(b)
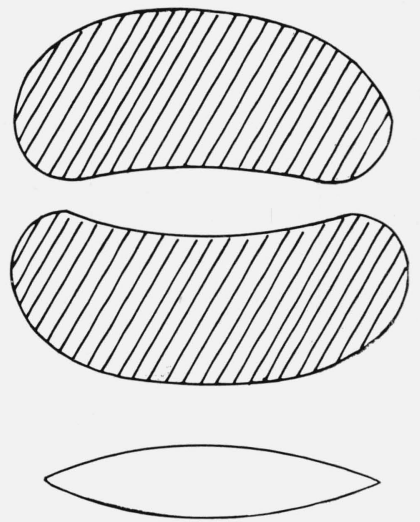

(c)

Figure 10. Lens-action geometry from multiply-connected electron density distributions.

a. A cusped lens, which does not possess a caustic even though it converges rays. b. A diverging lens.

c. A cusped lens which produces a caustic inside the focus.

Instead, it is useful to consider an interrelated configuration of electron density concentrations and rarefactions. The major difference between independent irregularities and interrelated concentrations is that in the first case there must exist discontinuities in the derivative of the total electron content from one point on the sky to another, while in the second case, the derivative of the electron distribution is continuous. Since the integrated content is nonzero and finite, there are points of inflection in the electron distribution, if it is continuous with more than one maximum. These specifications describe a wave structure. It remains to be seen whether wavelike isoelectronic surfaces produce the actual caustic behavior exhibited by the observed scintillations.

Figure 11 depicts an electron distribution having the correct properties. The wave trough between inflection points $B$ and $B^{\prime}$ focuses radio-star energy. Adjacent rays near the points of inflection bend by almost the same amount, since the curvature of the wave vanishes there by definition. The intersection points of rays at the edges of the trough therefore intersect on the opposite side of the central plane of the trough, and at great distances from it. Adjacent central rays intersect relatively close to the trough. The resultant caustic opens out away from the 


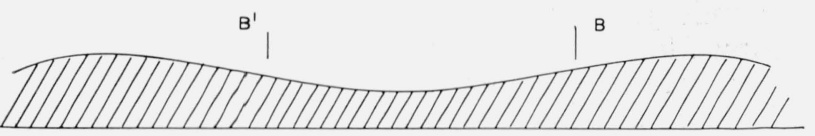

FIgURE 11. Waves in the isoelectron density surfaces show points of inflection in the derivative of the integrated electron density contours on the sky.

Between inflection points $B$ and $B^{\prime}$, the distribution acts as a converging lens with a caustic outside the focus.

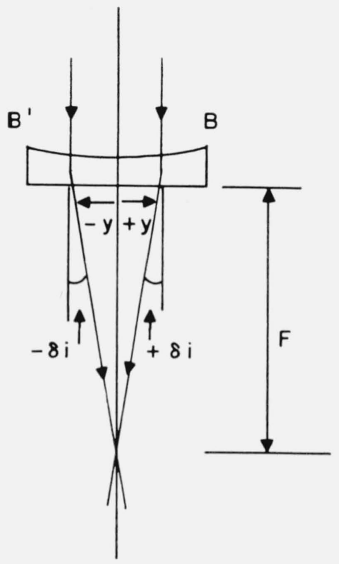

FIGURE 12. The detailed geometry of the focusing region shown in figure 11.

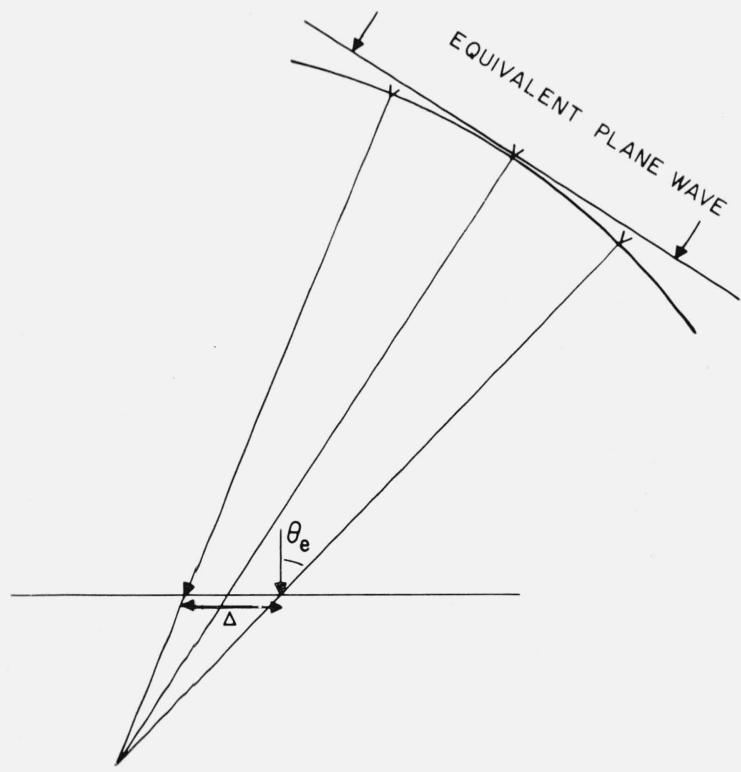

FIgure 13. A spherical wave received by a two-element interferometer is equivalent to a plane wave propagating along the bisector of the angle subtended by the baseline, $\Delta$, as seen from the center of the wave.

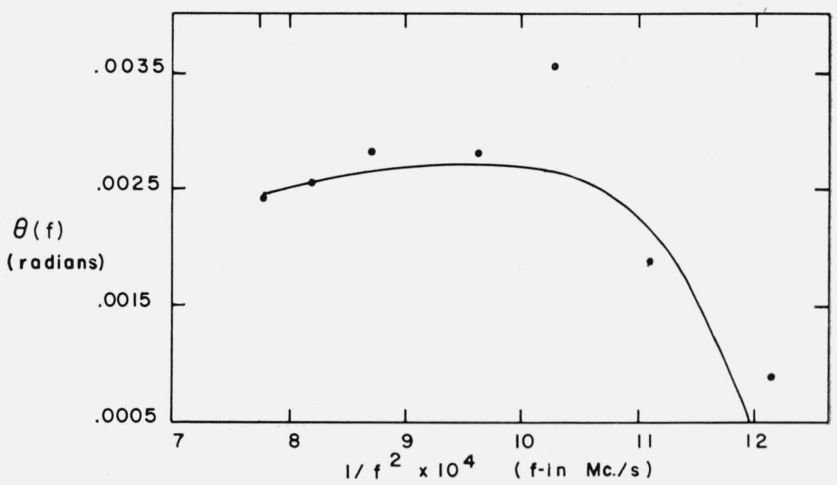

FIgURE 14. Departure of the source from its position at the midpoint of the scintillation of figure 1, as a function of the inverse square of the frequency.

The continuous curve represents the computed departure, the points, the observed departure. The technique of the computations is described in the text.

trough, and behaves like the forked scintillation.

Suppose that the upper surface of the wave of figure 11 is given by

$$
A=-A_{0} \cos 2 \pi y / \lambda .
$$

$A_{0}$ is the semi-amplitude of the wave, $y$, the coordinate along the wave measured from the trough, and $\lambda$, the wavelength measured from crest to crest. (2) assumes that the wave is similar to an ocean wave, with uniform density below a sinusoidal surface. This assumption does not represent a real restriction, as far as the interpretation of the spectrographic observations is concerned. The refractive deviation $\theta$ of a ray incident at angle $i$ onto a surface into which the dielectric constant, $\epsilon$, jumps by a small amount $\Delta \epsilon$ is, by an adaptation of Snell's law,

$$
\Theta \sim \frac{1}{2} \frac{\Delta \epsilon}{\epsilon} \tan i
$$

Substituting from (2) in (3), we find, when $\epsilon \sim 1$, that

$$
\Theta=\frac{1}{2} \Delta \epsilon\left(2 \pi A_{0} / \lambda\right) \sin 2 \pi y / \lambda .
$$

The focal length of the region at distance $|y|$ from the center of the trough is $F$, satisfying $y=F \Theta$ (see fig. 12). Substituting this in (4), we find

where

$$
F=(\lambda / 2 \pi L) y /(\sin 2 \pi y / \lambda),
$$

$$
L=\frac{1}{2} \Delta \epsilon A_{0}
$$

is the optical thickness of the equivalent converging lens. For central regions of the lens, $y \ll \lambda / 2 \pi$. Equation (5) then shows that $F=(\lambda / 2 \pi)^{2} / L$. This is the smallest focal length for any value of $y$ in the trough. Over a small central region of the lens, the focal length is independent of $y$. Without going into 
the details of the derivation, we shall merely state that the caustic curve for this lens is, when defined parametrically in terms of $y$,

$$
\begin{aligned}
& x_{1}=y-(\lambda / 2 \pi) \tan 2 \pi y / \lambda \\
& x_{2}=(\lambda / 2 \pi)^{2}[\sec 2 \pi y / \lambda] / L
\end{aligned}
$$

where $x_{1}, x_{2}$ are coordinates at right angles to, and along the optic axis (the center of the trough is on the line $x_{1}=0$ ).

The minimum duration $t_{\min }$ of the scintillation depends, among other things, on the "circle" of confusion at the "best" focus in the presence of the caustic. The first of (6) permits an estimate of the minimum duration on this basis. Suppose that the average ray impinging on the lens falls at $y= \pm \lambda / 8$, halfway from the center of the lens to its edge. The total average width of the caustic is

$$
2 \bar{x}_{1}=(\pi-2)(\lambda / 2 \pi) .
$$

The duration as a function of frequency during the scintillation shown in figure 1 was graphed in figure 8. The intercept on the axis $1 / f^{2}=0$ of the linear best fit to the data represents the time it takes for the trough of the ionospheric wave pattern to move past the observation point. The time is $142 \mathrm{sec}$, and represents $\lambda / 2 v$, where $v$ is the speed of the ionospheric wind. If our caustic explanation of the minimum duration is correct we should expect that

$$
\frac{2 \bar{x}_{1}}{t_{m}}=K \frac{(\pi-2) \lambda / 2 \pi}{39}=\frac{\lambda}{142},
$$

with $K=1$. Solution of (8) for $K$ indicates that $K=0.80$, satisfactorily close to unity and suggesting the essential correctness of our identification of the minimum duration with the total average width of the caustic. The estimate in (7) of the size of the circle of confusion resulting from spherical aberration may have been too large, by about one-fourth. That is, the actual minimum duration corresponds not to $y= \pm \lambda / 8$ but rather to $\pm \lambda / 10$.

\section{Distance to the Lens}

The distance to the lens follows from the apparent angular drift rate as a function of frequency. At frequencies very high compared with the frequency of best focus, the scintillation does not exist, and the radio star energy comes just from the normal direction as though the ionosphere were not present at all. The direction of arrival cannot vary as the ionospheric irregularity drifts by. At an intermediate frequency, the angle of arrival is approximately constant over the baseline, but changes as the lens drifts by. At or close to the focused frequency, rays arrive at an observing point from a wide section of the lens. Consider the lens as though it were perfect, and could bring all rays to the same point in space, either above or below the interferometer. On the high frequency side, the geometrical focal point lies underground, below the interferometer. Imagine yourself looking back towards the interferometer and ionosphere from this high frequency focus. The interferometer baseline subtends an angle that covers only part of the ionospheric lens. As the frequency decreases, the focal point moves up closer to the interferometer and the lens, as the angle subtended by the baseline covers a larger fraction of the lens. For some frequency greater than the observed focused frequency, the baseline covers the entire lens.

A perfect lens produces a spherical wave impinging on the interferometer. The interferometer measures direction of arrival in terms of the relative phase of the wave as it is received at the two ends of the baseline. Figure 13 shows that the spherical wave may be replaced by an equivalent plane wave, whose direction of arrival is along the bisector of the angle formed between rays arriving at the ends of the baseline. At the beginning of a scintillation, rays from the leading edge of the ionospheric lens will fall first on the windward end of the baseline. Until rays from the lens cover both ends of the baseline, the scintillation has not reached full intensity. At the moment both antennas intercept rays from the lens, the direction of arrival is the average between the extreme ray from the lens, and a ray closer to the center of the lens.

A rough measure of the direction of arrival of the extreme ray is given by the apparent direction of arrival of the rays at high frequency, say $f_{0}$, near the start of the scintillation. If rays arising from the edge of the effective lens bend at angle $\theta_{e}(f)$, the relation

$$
\theta_{e}(f)=\theta_{e}\left(f_{0}\right) f_{0}^{2} / f^{2}
$$

should hold. A first estimate of the extreme angle of arrival $\theta_{e}$ at any frequency $f$ follows from equating $\theta_{c}$ at some high frequency, $f_{0}$, to $\theta\left(f_{0}\right)$, the actual angle of arrival observed at the frequency $f_{0}$. At the start of a scintillation the difference between observed and extreme angles of arrival equals one-half of the angle subtended by the interferometer baseline as seen from the focal point. If an accurate method for determining the extreme angle of arrival were available, the observed angle of arrival would then permit finding just how far the focal point is below the surface of the earth.

Suppose that we know, or can estimate, the frequency at which the scintillation has its minimum duration. Since the duration as a function of frequency has a rather broad minimum this frequency is not well determined experimentally within a range of a megacycle or so. The frequency of this minimum is $f_{\min }$. At $f_{\min }$ the focal length of the lens is $F_{m}$. Now, the actual angle of arrival $\theta(f)$, observed at the onset of the scintillation, is less than the extreme angle coming from the edge of the lens. The difference is $\theta_{e}(f)-\theta(f)=1 / 2 \Delta /(F-a)$, where $\Delta$ is the baseline of the interferometer, and $a$ is the distance from the interferometer to the irregularity. Now, $F_{m}=a$; furthermore

$$
F(f)=F_{m} f^{2} / f_{\min }^{2}=a f^{2} / f_{\min }^{2} .
$$


Therefore,

$$
\Theta_{e}(f)-\Theta(f)=\left(\frac{1}{2} \Delta / a\right) /\left[\left(f^{2}-f_{\min }^{2}\right) / f_{\min }^{2}\right] .
$$

Since $f_{\min }$ is known only approximately, we assume its value at the start of the computation. Then, with an assumption on $\theta_{e}\left(f_{0}\right)$, and two observations of $\Theta(f)$ at different frequencies, we can iterate until both $\theta(f)$ values give the same determination of $a$. We can then compute $\theta(f)$ for all $f$ values, including in particular the high-frequency value, $f_{0}$. By iteration on the starting value of $\Theta_{e}$, which was assumed to be $\Theta_{e}\left(f_{0}\right)=\Theta\left(f_{0}\right)$, we then fit three observed values of $\Theta$ to a given set of values, $f_{\min }, a$, and $\Theta_{e}\left(f_{0}\right)$.

This procedure has been carried out for the one scintillation shown in figure 1 , and the results are shown in figure 14. As we expected, the observed displacement of the source shows a maximum at a frequency higher than the focus frequency. The curve of the frequency-dependent displacement computed from (11) is also shown. Actually instead of following the procedure outlined above, in which the computed displacement perforce passes through three of the observed points, we attempted to make the curve fit the overall trend of the observed points as well as possible, with what success the reader may judge. The solution is very sensitive to the value of $f_{\mathrm{mln}}$, which turned out to be $27.66 \mathrm{Mc} / \mathrm{s}$. This seems in reasonable agreement with the appearance of the scintillation itself, as shown in figure 1. The corresponding value of $a=347 \mathrm{~km}$. This value depends critically on the exact shape of the angular displacement as a function of frequency. If, for example, the computed curve is made to pass through the last two points, at low frequencies just a bit higher than the focused frequency, the value of $a=$ $500 \mathrm{~km}$. But, in that case, the computed curve falls below all of the observed points except the one at high frequency through which it is forced to pass. In other words, in order for the computed curve to show the steep fall-off to zero displacement just above the focused frequency it is necessary that $a$ have the smaller value described above.

The height above ground corresponding to the slant range $a=347 \mathrm{~km}$ is $178 \mathrm{~km}$. At the focus, the value of $\theta_{e}\left(f_{\mathrm{min}}\right)=0.00481$ radians. This is half of the angle subtended by the lens, and should be increased by about 25 percent to allow for the caustic effect mentioned earlier. At the range $a$, the resulting wavelength, $\lambda=17 \mathrm{~km}$. Since, as can be seen from figures 1,2 , and 3 , there is a tendency for the scintillations to occur once every few minutes, say, about $300 \mathrm{sec}$, the speed $v=17 \mathrm{~km} / 300 \mathrm{sec} \sim$ $60 \mathrm{~m} / \mathrm{sec}$. Alternatively, we can compute $v$ from (8) : $v=1 / 2 \times 17 \mathrm{~km} / 142 \mathrm{sec} \sim 60 \mathrm{~ms}$. At $347 \mathrm{~km}$, the Fresnel zone radius is $R=\sqrt{\lambda a} \sim 2 \mathrm{~km}$. Since this value is decidedly smaller than the focusing structure observed, we conclude that diffractive effects are relatively unimportant, as indeed we have assumed them to be.

One last parameter can be found from the theory, now that $\lambda$ is known. From (5), with $2 \pi y / \lambda=\pi / 2$, we find that $347 \mathrm{~km}=\lambda^{2} / 8 \pi L$. Solving for $L$, with $\lambda=17 \mathrm{~km}$, we find $L=33 \mathrm{~m}$. If, as may be plausible, we set $A_{0} \sim \lambda$, then $\Delta \epsilon=3 \times 10^{-3}$. Since $\epsilon \sim 1$, we conclude that the wave involved in these observations could be produced by an electron discontinuity of only 0.3 percent, maintained over a height range of $17 \mathrm{~km}$.

\section{General Structure of Broadband Scintillations}

Although large numbers of scintillations appearing on our records show at least some of the characteristics of the particular scintillations described above, it is not true that all do. The most common feature described above is the broadband characteristic, shown by the great majority of scintillations. Next, is the angular displacement pattern as a function of frequency, which scintillations show strongly only in some particular regions of the sky, and then not in all cases. It is not our objective here to discuss the systematics of all scintillations, but rather to detail what can be found out intensively from just one case. However, we should point out how simply broadband scintillations may be consistent with our conclusions even though the remaining phenomena described above do not appear.

For example, the fork effect observed at low frequencies, and interpreted above in terms of points of inflection on the distribution of electrons in ionospheric waves, might be obliterated if there existed a smaller scale structure destroying the zero-curvature condition essential to our explanation of the forks. Also, the existence of a clear focus depends on the quality of the lens, especially on its freedom from spherical aberration, and on the angular size of the radio star and the angle at which it is viewed. In addition the existence of fine structure on the basic wave pattern has an effect similar to spherical aberrations in destroying the focus. The fact of a broadband scintillation implies a focus in an approximate sense. If, additionally, random angular deviations occur on a scale smaller than the systematic deviations that produced the broadband increase in power they will diffuse the sharpness of the focus, and decrease the clarity of angular shifts as a function of frequency.

To expand on this last consideration, we note that for irregularities one order of magnitude smaller than we have found, the Fresnel zone is larger than the irregularity. The Fresnel zone can be thought of in two different ways: it defines the area of a plane surface from within which waves arrive at the observing point without path differences exceeding one-half wavelength; or, more than one irregularity contributes to the total wave field at a point on the ground when the irregularities are smaller than the Fresnel zone. For these small irregularities, the angles of arrival then depend on $c / \lambda f$, where $c$ is the speed of light. Suppose that there exists a complete spectrum of irregularity sizes, including the very large ones that we have discussed in this report. Suppose that angular deviations resulting from diffraction at the large irregularities are small 
compared to the diffractive deviations from small irregularities at some high frequency. As the frequency is decreased, the refractive deviations increase, as the inverse square of the operating frequency. On the other hand, diffractive deviations increase only as the inverse frequency. At some low frequency the large irregularities first, and then at some still lower frequency in addition the small irregularities (still large compared to the wavelength), go over into a regime where refractive effects are dominant.

In this sense, we consider that the presence or absence of fine focus, systematic angle-of-arrival effects, and forks measure the presence or absence of fine structure in the ionosphere. In any case, the broadband nature of the scintillations indicates large scale structure. Only with a close look into the data is it possible to reveal the presence there also of smaller structures.

\section{References}

Boischot, A., R. H. Lee, and J. W. Warwick (1960), Lowfrequency solar bursts and noise storms, Astrophys. J. 131, $61-67$.

Booker, H. G. (1958), The use of radio stars to study irregular refraction of radio waves in the ionosphere, Proc. IRE 46, 298-314.

Lawrence, R. S., J. L. Jespersen, and R. C. Lamb (1961), Amplitude and angular scintillations of the radio source Cygnus-A observed at Boulder, Colo. J. Res. NBS 65D (Radio Prop.), No. 4, 333-350.

Lee, R. H., and J. W. Warwick (1964), A Spectrographic Interferometer, (to be published).

Little, C. G., G. C. Reid, E. Stiltner, and R. P. Merritt (1962), An experimental investigation of the scintillation of radio stars observed at frequencies of 223 and 456 megacycles per second from a location close to the auroral zone, J. Geophys. Res. 6\%, 1763-1784.

Warwick, J. W. (1961), Theory of Jupiter's decametric emission, Annals New York Academy of Sciences, 95, 39-60.

Warwick, J. W. (1963) Dynamic spectra of Jupiter's decametric emission, 1961, Astrophys, J. 13\%, 41-60.

Wild, J. P., and J. A. Roberts (1956), The spectrum of radiostar scintillations and the nature of inequalities in the ionosphere, J. Atmospheric Terrest. Phys. 8, 55-75.

(Paper 68D2-331) 